\title{
On the metallicity of open clusters
}

\section{Photometry ${ }^{\star}$}

\author{
E. Paunzen ${ }^{1}$, U. Heiter ${ }^{2}$, M. Netopil ${ }^{1,3}$, and C. Soubiran ${ }^{4}$ \\ 1 Institut für Astronomie der Universität Wien, Türkenschanzstr. 17, 1180 Wien, Austria \\ e-mail: Ernst.Paunzen@univie.ac.at \\ 2 Department of Physics and Astronomy, Uppsala University, Box 515, 75120 Uppsala, Sweden \\ 3 Hvar Observatory, Faculty of Geodesy, University of Zagreb, Kačićeva 26, 10000 Zagreb, Croatia \\ ${ }^{4}$ Université Bordeaux 1, CNRS, Laboratoire d'Astrophysique de Bordeaux, BP 89, 33270 Floirac, France
}

Received 25 January 2010 / Accepted 22 March 2010

\section{ABSTRACT}

\begin{abstract}
Context. Metallicity is one of four free parameters typically considered when fitting isochrones to the cluster sequence. Unfortunately, this parameter is often ignored or assumed to be solar in most papers. Hence an unknown bias is introduced in the estimation of the other three cluster parameters (age, reddening and distance). Furthermore, studying the metallicity of open clusters allows us not only to derive the Galactic abundance gradient on a global scale, but also to trace the local solar environment in more detail.

Aims. In a series of three papers, we investigate the current status of published metallicities for open clusters from widely different photometric and spectroscopic methods. A detailed comparison of the results allows us to establish more reliable photometric calibrations and corrections for isochrone fitting techniques. Well established databases such as WEBDA help us to perform a homogeneous analysis of available measurements for a significant number of open clusters.

Methods. The literature was searched for $[\mathrm{Fe} / \mathrm{H}]$ estimates on the basis of photometric calibrations in any available filter system. On the basis of results published by Tadross, we demonstrate the caveats of the calibration choice and its possible impact. In total, we find 406 individual metallicity values for 188 open clusters within 64 publications. The values were, finally, unweightedly averaged. Results. Our final sample includes $[\mathrm{Fe} / \mathrm{H}]$ values for 188 open clusters. Tracing the solar environment within $4000 \times 4000 \mathrm{pc}^{2}$ we identify a patchy metallicity distribution as an extension to the Local Bubble that significantly influences the estimation of the Galactic metallicity gradient, even on a global scale. In addition, further investigations of more distant open clusters are clearly needed to obtain a more profound picture at Galactocentric distances beyond $10000 \mathrm{pc}$.

Conclusions. Only a combination of all available photometric and spectroscopic data will shed more light on how the local and global Galactic properties are correlated with metallicity.
\end{abstract}

Key words. Galaxy: abundances - open clusters and associations: general - stars: abundances

\section{Introduction}

One of the most important key parameters for our understanding of stellar formation and evolution, is the intrinsic metallicity of (proto-)stars of a given mass. Even in the early stages of stellar evolution, the metallicity severely influences the cooling and collapse of ionized gas (Jappsen et al. 2007). By comparing detailed simulations with observations, it has been shown that clouds of lower metallicity have a higher probability of fragmentation, indicating that the binary frequency is a decreasing function of the cloud metallicity (Machida 2008).

Looking at the global properties of our Milky Way, a radial metallicity gradient throughout the Galactic disk was discovered several decades ago, which provides strong constraints on the mechanism of galaxy formation. Models now show that the stellar formation as a function of Galactocentric distance strongly influence the appearance and the development of the metallicity gradients (Chiappini et al. 2001). Our knowledge is based on stellar data, for example those of Cepheids (Cescutti et al. 2007), or open clusters (Chen et al. 2003) as well as globular clusters (Yong et al. 2008). However, these approaches are still

^ Tables 1 and 3 are only available in electronic form at http//wWW . aanda. org not satisfactory. All individual stellar estimates are limited to the accurate distance estimation of field stars and the uncertainties in the spectroscopic abundance analysis for very distant and therefore faint objects. The metallicity compilations of open clusters (for example Chen et al. 2003) are normally based on inhomogeneous data sets.

In a series of papers, we concentrate on the metallicity of open clusters using the results of various techniques and methods. Our final goals are 1) to derive homogeneous metallicities of all available and published data; 2) to establish a more robust photometric calibration on the basis of various filter systems; and 3 ) to investigate the influence of metallicity on isochrone fitting techniques. There have been several studies (e.g., Twarog et al. 1997; Magrini et al. 2009) of this nature, but none have taken advantage of all available photometric as well as spectroscopic data.

Besides the investigation of the global Galactic properties, we are also able to shed more light on the validity of the automatic open cluster parameter estimations performed by Kharchenko et al. (2005). Their estimation of the age, reddening and distance of 650 open clusters and the follow-up conclusions (Kharchenko et al. 2009) are all based on solar metallicity. However, the classical technique of isochrone fitting in various 
colour-magnitude diagrams, also incorporates the metallicity as an a-priori free parameter.

We present our extensive investigation in a series of three papers divided into a photometric, a spectroscopic, and a "calibration" part.

\section{Target selection and literature assessment}

Metallicities, for photometric observations, are often listed either as $[\mathrm{Fe} / \mathrm{H}]$ or $Z$ values. If not stated otherwise, these parameters can be transformed using the helium-to-metal enrichment relation $Y=0.23+2.25 \times Z$ and a solar value $Z=0.019$, as given in Sect. 2.1 of Girardi et al. (2000). Almost all available photometric calibrations derive $[\mathrm{Fe} / \mathrm{H}]$ as the default standard parameter for the metallicity. This is because the iron lines, besides hydrogen and helium lines, dominate the optical spectrum for a wide variety of main sequence stars. Therefore, if integrating over one optical filter, the abundance of iron can be used as some kind of standard candle. Another advantage to using this element is that it is normally unaffected by the main sequence evolution.

The metallicity determinations obtained on the basis of isochrone fitting only, were not taken into consideration because the grid of isochrones are normally only sparsely available for the $Z$ parameter (Schaller et al. 1992). Therefore, authors only consider whether the isochrones with a $Z$ value higher or lower than that of the Sun, fit the observations more closely. One typical example is the paper by Piatti et al. (2006) who published $Z=0.040$, corresponding to $[\mathrm{Fe} / \mathrm{H}]=+0.37 \mathrm{dex}$, for the open cluster NGC 5288. Unfortunately, they do not include a corresponding plot with isochrones for the different metallicities.

We searched the literature for metallicity estimates of open cluster members on the basis of photometric calibrations using $\mathrm{WEBDA}^{1}$ as a starting point. The apparent double clusters NGC $2451 \mathrm{~A} / \mathrm{B}$ were excluded from our investigation because there is an unsolved and constant confusion about the true nature of these aggregates (Platais et al. 2001).

In total, we found 406 individual metallicity values for 188 open clusters in 64 publications. Table 1 lists the values and the number of stars used to derive the metallicities, if available, and the employed photometric filter systems, which are:

- Caby: Strömgren and the Ca filters measuring the Ca II HK lines (Anthony-Twarog et al. 1991);

- DDO: David Dunlap Observatory system (McClure \& van den Bergh 1968);

- Johnson: UBVRI colors (Bessell 1995);

- Strömgren: uvby $\beta$ photometry (Strömgren 1966);

- Vilnius: a seven filter system (Smriglio et al. 1990);

- Washington: a four filter system introduced by Canterna (1976).

We note that open clusters are investigated in all major photometric systems apart from the Geneva one. However, in the Geneva 7-color photometric system, blanketing sensitive indices are available (Paunzen et al. 2005), which could be used as a metallicity indicator.

The cluster parameters (age, reddening, and distance) were taken from Paunzen \& Netopil (2006). The only exceptions are Loden 807, NGC 7209, Ruprecht 20, and Ruprecht 32, for which the values were taken from the updated list of Dias et al. (2002) as well as Berkeley 1 and Ruprecht 46, for which the parameters

1 http://www . univie.ac .at/webda from WEBDA were used. The distance from the Galactic centre was calculated in the standard way using $8 \mathrm{kpc}$ for the Sun (Groenewegen et al. 2008). An error propagation was applied assuming that the uncertainties in the Galactic coordinates for the individual open clusters are negligible.

As a next step, we compared the results of two references using identical data sets, but different photometric calibrations.

\section{A test case from the literature}

The pitfalls of using two different photometric calibrations for identical data sets are clearly illustrated in the papers by Tadross (2001, 2003).

This author performed robust derivations of the cluster age, reddening, distance, and metallicity on the basis of Johnson $U B V$ photometry. In the first paper, he used average metallicity values derived from the calibrations of Carney (1979) and Cameron (1985a), whereas in the second, only values from Cameron (1985a), were applied (Tadross, private communication). The second study was found to provide far more reliable results compared to the literature.

Both calibrations are based on the normalized ultraviolet excess $\delta(U-B)_{0.6}$, introduced by Sandage (1969), which is compared to spectroscopically determined elemental abundances. While Carney (1979) used a list of published abundances of subdwarfs, Cameron (1985a) constructed a grid consisting of blanketing lines and lines of constant metallicity based on theoretical as well as empirical data. The determined excess values were then transformed to $[\mathrm{Fe} / \mathrm{H}]$ using a second order polynomial in both references.

Figure 1 compares the $[\mathrm{Fe} / \mathrm{H}]$ values from both Tadross papers. It is obvious that there are seventeen clusters with values of less than -1.0 dex and seven with less than -1.5 dex in Tadross (2001), whereas there is only one cluster with $[\mathrm{Fe} / \mathrm{H}]$ less than -1.0 dex in Tadross (2003). We investigated the plausibility of these cases of low metallicity. Using data of Cepheids, Pedicelli et al. (2009) showed that $[\mathrm{Fe} / \mathrm{H}]$ values significant below -0.5 dex are not expected in the Galactic disk where open clusters are located, even at distances of $17 \mathrm{kpc}$. The compilation of open cluster data by Chen et al. (2003) includes only three aggregates with $-0.5>[\mathrm{Fe} / \mathrm{H}]>-1.0 \mathrm{dex}$, which is compatible with the results by Magrini et al. (2009).

In Table 2, we list the seven extreme cases together with the cluster parameters based on the list by Paunzen \& Netopil (2006). For two clusters, Berkeley 7 and Berkeley 30, respectively, the $[\mathrm{Fe} / \mathrm{H}]$ determinations from Tadross (2003) seem to be well in the expected range. For the remaining five clusters, the new determinations are still in the range $-0.46>[\mathrm{Fe} / \mathrm{H}]>-0.85 \mathrm{dex}$, which is surprisingly low for the given ages and distances of the aggregates. For Berkeley 60, a large error in the distance and reddening from the literature is evident. Ann et al. (2002) list a near solar metallicity (+0.07 dex) for this cluster based on isochrone fitting to Johnson UBVI measurements. For NGC 581 (Sanner et al. 1999) and NGC 2244 (Bonatto \& Bica 2009), we also find a published solar metallicity. The cluster NGC 1750 is very interesting in the sense that it overlaps with NGC 1758 (Straižys et al. 2003). Therefore the choice of the members is essential to derive correct values. In summary, we find no evidence that the low metallicities are supported by any other source.

The remaining values (Fig. 1) are within a range of $+0.3>[\mathrm{Fe} / \mathrm{H}]>-1.0 \mathrm{dex}$, which is the expected one for open clusters (Chen et al. 2003). For the following analysis, we intend to use the discussed metallicities in a proper way because 
E. Paunzen et al.: On the metallicity of open clusters. I.

Table 2. The open clusters with very low $[\mathrm{Fe} / \mathrm{H}]$ estimates from Tadross (2001) and the comparison with those from Tadross (2003).

\begin{tabular}{|c|c|c|c|c|c|c|c|c|c|c|c|c|}
\hline Cluster & & $\begin{array}{c}{[\mathrm{Fe} / \mathrm{H}]_{01}} \\
{[\mathrm{dex}]}\end{array}$ & $\begin{array}{c}{[\mathrm{Fe} / \mathrm{H}]_{03}} \\
{[\mathrm{dex}]}\end{array}$ & $\begin{array}{c}d \\
{[\mathrm{pc}]}\end{array}$ & $\begin{array}{c}\sigma \\
{[\mathrm{pc}]}\end{array}$ & $N$ & $\begin{array}{c}E(B-V) \\
{[\mathrm{mag}]}\end{array}$ & $\begin{array}{c}\sigma \\
{[\mathrm{mag}]}\end{array}$ & $N$ & $\begin{array}{c}\text { Age } \\
{[\mathrm{Myr}]}\end{array}$ & $\begin{array}{c}\sigma \\
{[\mathrm{Myr}]}\end{array}$ & $N$ \\
\hline Berkeley 7 & $\mathrm{C} 0150+621$ & -1.75 & -0.25 & 2509 & 195 & 3 & 0.80 & 0.00 & 3 & 4 & 1 & 3 \\
\hline Berkeley 30 & $\mathrm{C} 0655+032$ & -2.67 & +0.10 & 3990 & 1140 & 5 & 0.50 & 0.07 & 5 & 565 & 350 & 5 \\
\hline Berkeley 60 & $\mathrm{C} 0015+606$ & -2.26 & -0.77 & 3245 & 1634 & 2 & 0.62 & 0.35 & 2 & 160 & 3 & 2 \\
\hline Berkeley 62 & $\mathrm{C} 0057+636$ & -2.39 & -0.85 & 2063 & 400 & 6 & 0.84 & 0.04 & 6 & 17 & 12 & 6 \\
\hline NGC 581 & C0129+604 & -1.54 & -0.85 & 2569 & 229 & 9 & 0.41 & 0.03 & 10 & 21 & 9 & 11 \\
\hline NGC 1750 & $\mathrm{C} 0500+235$ & -1.97 & -0.69 & 646 & 20 & 3 & 0.34 & 0.01 & 3 & 217 & 30 & 3 \\
\hline NGC 2244 & $\mathrm{C} 0629+049$ & -1.77 & -0.46 & 1595 & 119 & 9 & 0.46 & 0.03 & 10 & 5 & 2 & 10 \\
\hline
\end{tabular}

Notes. The cluster parameters are based on the list by Paunzen \& Netopil (2006).

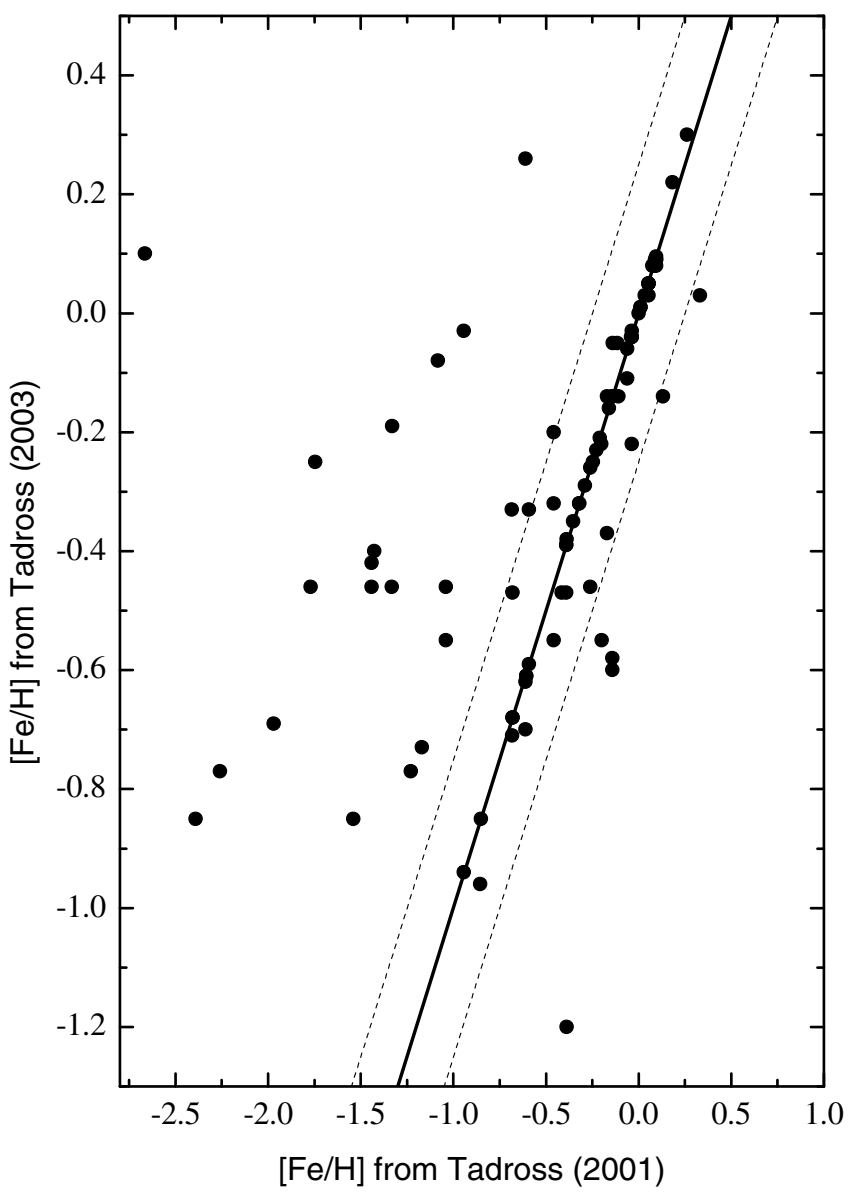

Fig. 1. A comparison of the $[\mathrm{Fe} / \mathrm{H}]$ values derived via two different photometric calibrations as published by Tadross $(2001,2003)$. The dashed lines represent \pm 0.25 dex offsets. Note the wide range of apparent underabundant aggregates in the first paper.

they fill an important gap within the published investigations. Because the later paper by Tadross supersedes the previous one, we considered only the values from Tadross (2003) for our further analysis.

\section{Mean $[\mathrm{Fe} / \mathrm{H}]$ metallicities and analysis}

The basic metallicity calibrations for the individual photometric systems are, in general, very similar to each other. The starting point is a set of so-called "standard objects" for which the metallicity is clearly determined. The effective temperature and the surface gravity are normally not used in the calibration process.
As a next step, a metallicity sensitive index within a specific photometric system has to be traced and correlated with the standard set of objects. The indices used are listed, in more details, below. There are only very few examples in which the whole variety of available photometric indices are used to correlate the metallicity. Martell \& Laughlin (2002) presented third order polynomials of Strömgren $b-y, m_{1}$, and $c_{1}$ with the complete permutation of these indices resulting in 20 coefficients with a listed precision of six digits. However, these heuristic calibrations are not widely used.

The metallicity values presented in Table 1 are based on various photometric calibrations within six photometric systems listed in Sect. 2. These calibrations are all based on the individual indices described in the following.

Caby: the $h k_{0}$ index measures the Ca II HK line strengths and thus the abundances (Anthony-Twarog et al. 1991). The values may be correlated with $[\mathrm{Fe} / \mathrm{H}]$ depending on the temperature sensitive index $(b-y)$.

$D D O$ : the $\delta C N$ measures the strength of the CN $4216 \AA$ anomaly for red giants (Janes 1975). The position of an object in the $C(45-48)$ versus $C(42-45)$ diagram is an indicator of the luminosity for solar metallicity. This location is used to predict the expected value of $C(41-42)$, a luminosity-dependent metallicity indicator. The $\delta C N$ is then the difference between the observed and predicted value that can be transformed via a linear relation to $[\mathrm{Fe} / \mathrm{H}]$. For example, in the series of papers by Clariá and coworkers, the calibration of Clariá \& Lapasset (1983) is used until 1991. From 1994 on, the calibration of Piatti et al. (1993) is used, based on a new definition of the cyanogen anomaly, now designated $\triangle \mathrm{CN}$. A comparative test published by Clariá et al. (2003, Ref. [21] in Table 1) indicates that the new calibration can result in a metallicity that differs by up to 0.1 dex from the previous one.

Johnson: the normalized ultraviolet excess, $\delta(U-B)_{0.6}$, is defined and calibrated relative to the Hyades main sequence in the two colour $(U-B)$ versus $(B-V)$ diagram. It is zero at $(B-V)=0.6 \mathrm{mag}$ for the metallicity of the Hyades, which is higher than solar, and sensitive to the effect of line blanketing (Sandage 1969).

Strömgren: the $m_{1}$ index is the standard line blanketing indicator for almost the complete temperature range (Strömgren 1966).

Vilnius: $(P-X)$ can be directly correlated with $[\mathrm{Fe} / \mathrm{H}]$ for cool-type stars later than G0 (Straižys \& Bartkevičius 1982).

Washington: starting with the solar-abundance relations $M-$ $T_{1}$ versus $C-M$ and using $T_{1}-T_{2}$ as a temperature sensitive index, the blanketing in the $M-T_{1}$ index is correlated with $[\mathrm{Fe} / \mathrm{H}]$. The blanketing in the $C-M$ index is correlated with the CN $4216 \AA$ anomaly of giants (Canterna 1976). Geisler et al. (1991) published a new empirical abundance calibration for the 


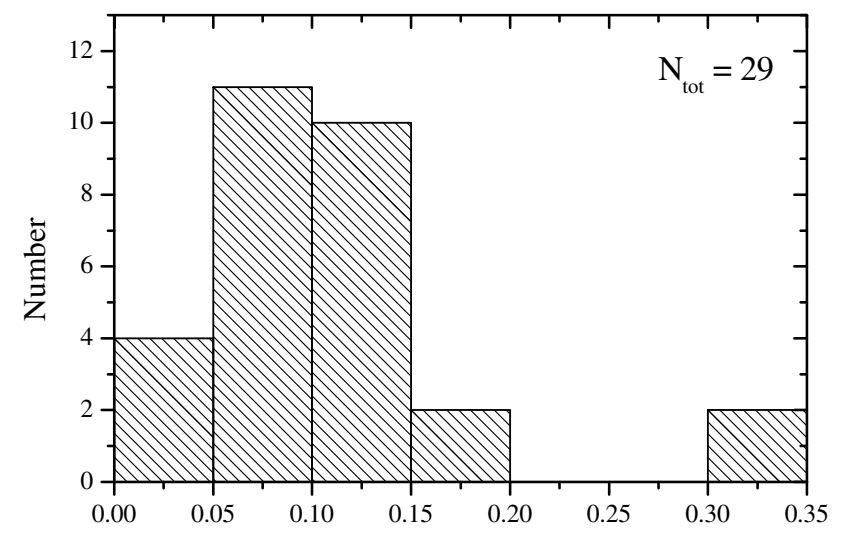

Error of the mean metallicity estimates [dex]

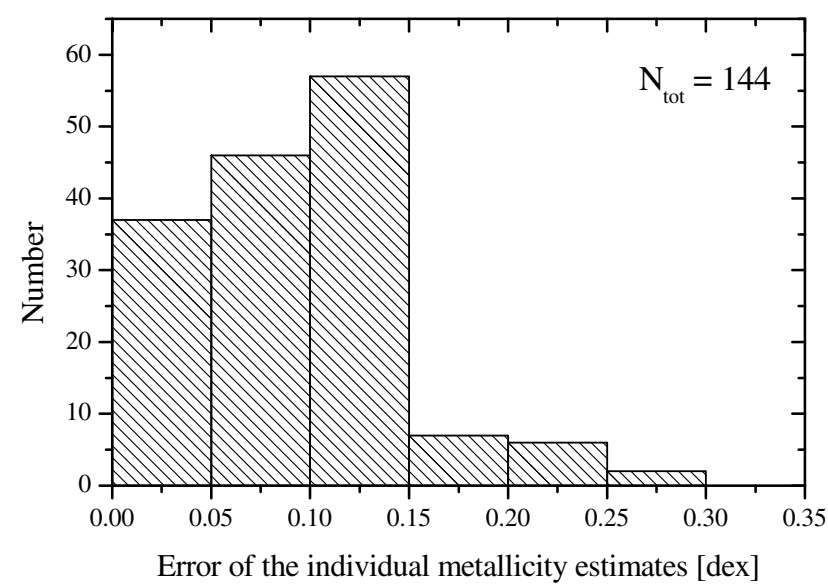

Fig. 2. The error distribution of the individual measurements and means for open clusters with $N \geq 4$ estimates from the literature.

Washington system, based on five metallicity-sensitive indices, which were used by all subsequent references given in Table 1.

The standard procedure for deriving the metallicity of a cluster is always very similar. After a proper selection of cluster members, which is especially important in studies using a rather limited number of stars (e.g. red giants), the objects have to be dereddened. In principle, the dereddening procedure has to be applied individually for each star rather than using a mean value, to take into account a possible differential reddening. Finally, using the respective metallicity calibrations applied to all selected objects, a mean value for the aggregate is calculated.

The values from the literature listed in Table 1 were averaged and the standard deviation of the mean was calculated. If only one value was available, the error from the literature was taken. If there is none available, we set the error to $0.1 \mathrm{dex}$, respectively. No weights were introduced in our procedure. We note that the metallicities are on a logarithmic scale, so a plain averaging results in a geometric and not in an arithmetic mean.

In total, $[\mathrm{Fe} / \mathrm{H}]$ values for 188 open clusters listed in Table 3 are available for our further analysis, among which 88 have more than one measurement available. The metallicities are comparable to those found for field Population I type stars in the vicinity of the Sun.

To test whether the listed errors in the individual metallicity estimates are realistic, we compared them to the errors in the means for open clusters with $N \geq 4$ available values. The latter choice guarantees that no outliers influence the results. In total, 29 open clusters with 144 individual measurements are used. Figure 2 shows the error distributions for these two samples. We

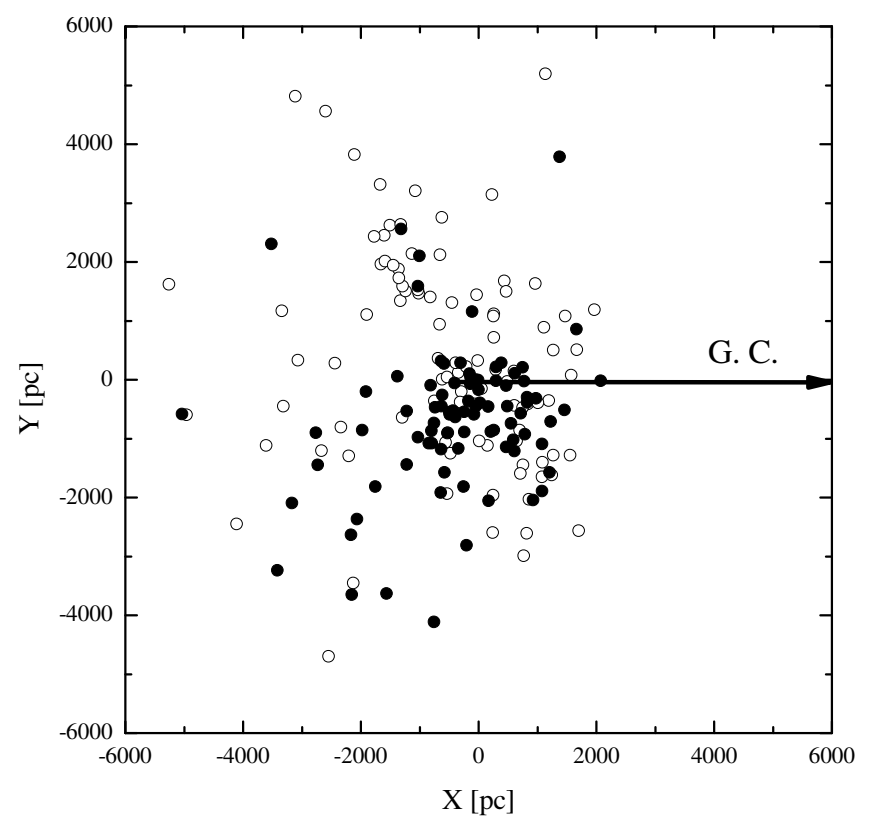

Fig. 3. The Galactic distribution of the final open cluster sample. We excluded Berkeley 22, Berkeley 29, and Tombaugh 2 because they are located far away from the Sun with a very large distance error. The open circles mark open clusters with only one available metallicity measurement whereas filled ones are those with more than one value. The Galactic centre is located at [+8000:0].

find that $90 \%$ of all values are below 0.15 dex for both samples. We therefore conclude that the individual errors are well within the range of those for the means, and that we do not introduce any bias by calculating the averages from the literature. There are, however, a few cases where the errors of the individual measurements might have been underestimated, notably NGC 2660 and NGC 6791.

Two of the most interesting and well studied topics are the metallicity distribution in the vicinity of the Sun and the Galactic metallicity gradient. With the current data set, we looked at both issues in more detail.

The Galactic distribution within the $[X: Y]$ plane is shown in Fig. 3. The Galactic centre is located at $[+8000: 0]$. The open clusters Berkeley 22, Berkeley 29, and Tombaugh 2 are excluded because they are located far away from the Sun with a very large distance error. The distribution of available metallicity values is not homogeneous within the whole area. The contours were therefore interpolated in a 3D manner using cubic splines. From the plot, it is obvious that the vicinity of $4000 \times 4000 \mathrm{pc}^{2}$ can be traced quite reliably. This is an excellent extension to similar efforts for the Local Bubble. Lallement et al. (2003) present maps of the density distribution of cold gas within $\pm 250 \mathrm{pc}$ from the Sun (supplemented by a small number of more distant observations) and find a radius for the Local Bubble of $60 \mathrm{pc}$, with extensions of up to $250 \mathrm{pc}$ and narrow "interstellar tunnels" out to at least $500 \mathrm{pc}$ (Welsh \& Shelton 2009). Figure 4 shows a contour plot of the metallicity distribution using our derived mean $[\mathrm{Fe} / \mathrm{H}]$ values. The patchy structure and the length scale resemble those of the Local Bubble very well. They continue out to 2000 pc from the Sun. On the other hand, Luck et al. (2006), investigated a far more extended region of $20000 \times 10000 \mathrm{pc}^{2}$, derived from abundances of 54 Cepheids. Their resolution is lower than ours but the general trends are comparable.

The metallicity gradient of the Milky Way has been the subject of detailed investigation. Observational data of not only star 


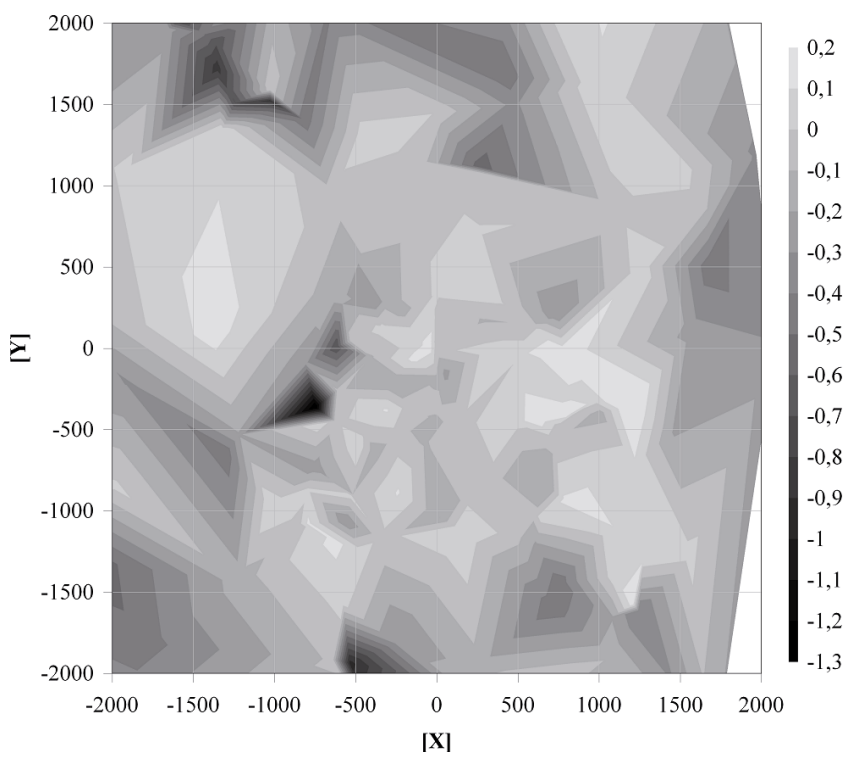

Fig. 4. A contour plot of the metallicity distribution within $4000 \times$ $4000 \mathrm{pc}^{2}$ around the Sun in the $[X: Y]$ plane. The patchy structure is consistent with results for the Local Bubble which has a diameter of up to $500 \mathrm{pc}$.

clusters, but also different star groups such as Cepheids and Population II type stars have been used to place constraints on Galactic evolutionary models. The published metallicity gradients differ widely. Pedicelli et al. (2009), for example, concluded that there are two different gradients for the inner and outer Galactic disc, respectively. Figure 5 shows the $[\mathrm{Fe} / \mathrm{H}]$ abundances versus the Galactocentric distance for our sample. The data are consistent with a rather wide range of metallicity gradients $\left(0.1\right.$ to $\left.0.3 \mathrm{dex} \mathrm{kpc}^{-1}\right)$. The estimate depends crucially on the data points with $R_{\mathrm{GC}}>10 \mathrm{kpc}$. But there is an apparent lack of open clusters in this region with more than one metallicity estimate. Keeping in mind the patchy structure of the local environment, the metallicity gradient should be estimated for different lines of sight within the Galactic plane. This analysis has, to our knowledge, never been done. We will tackle this topic in the final paper of this series.

\section{Conclusions and outlook}

We have presented our plans to complete a critical assessment of open cluster metallicities. In the first step presented here, we have collected all metallicity determinations based on photometric calibrations.

In total, 406 published values for 188 open clusters in 64 references were found that were averaged not taking into account any weights. A comparison of the papers by Tadross (2001, 2003), which are based on the same data sets but different applied calibrations, demonstrate how crucial the choice of the calibration is. The results, sometimes, widely diverge, in the sense that too low metallicities are derived.

Using published cluster parameters, we investigated the metallicity distribution in the vicinity of the Sun and the Galactic metallicity gradient in more detail. We are able to confirm and extend the patchy structure of the Local Bubble out to $2000 \mathrm{pc}$ from the Sun. The current data set does not allow us to draw any further conclusions about more distant Galactic regions. For the Galactic metallicity gradient, no conclusive value can be estimated with the current data set, because of the patchy local

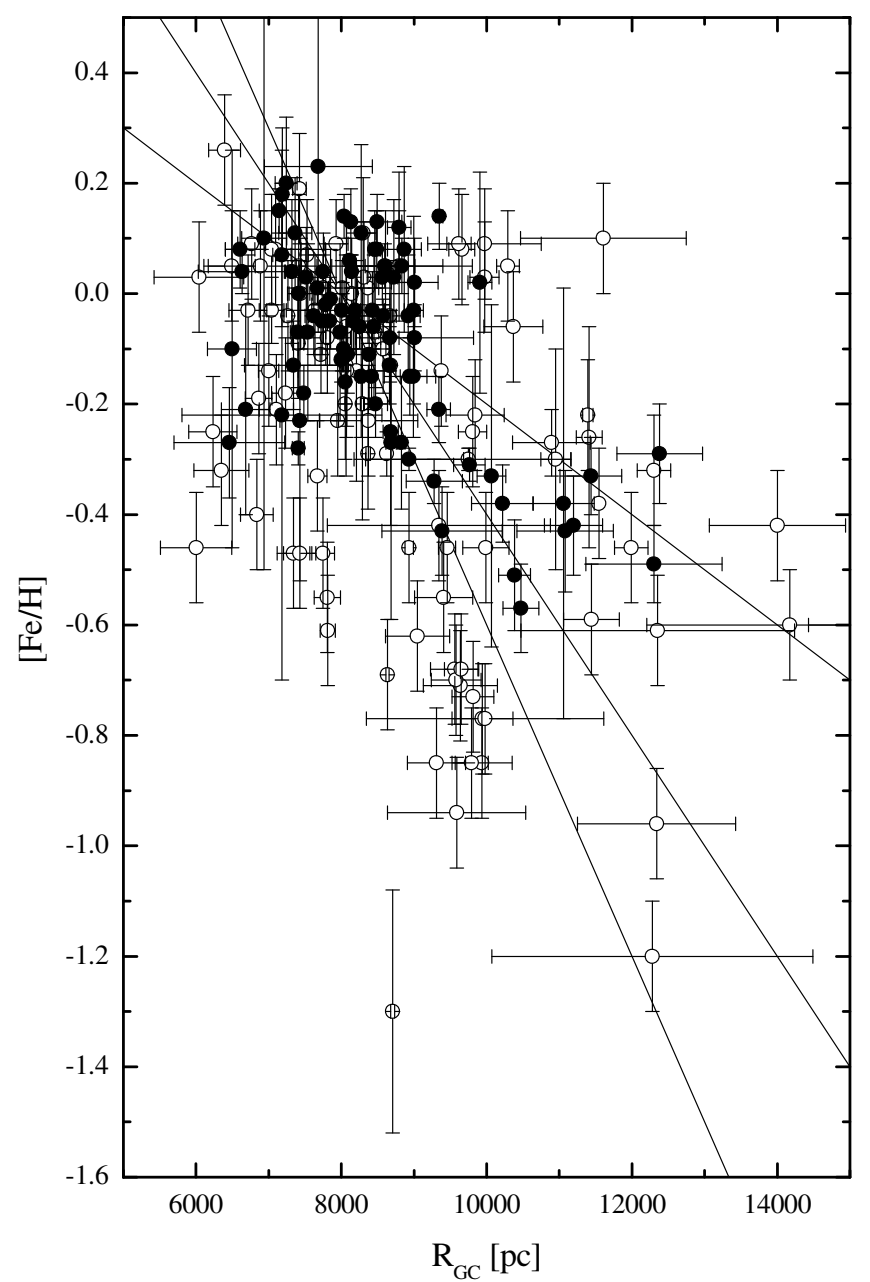

Fig. 5. The $[\mathrm{Fe} / \mathrm{H}]$ values versus the Galactocentric distance for our sample. The straight lines represent three different metallicity gradients of $0.1,0.2$, and $0.3 \mathrm{dex} \mathrm{kpc}^{-1}$, respectively. The Sun is at $R_{\mathrm{GC}}=8 \mathrm{kpc}$. The open circles mark open clusters with only one available metallicity measurement, whereas filled ones are those with more than one value.

distribution and the lack of very distant open clusters. Both topics will be investigated in forthcoming studies.

On the basis of the current sample, we propose to search for and analyse elemental abundances of open cluster members. A detailed comparison of the photometric and spectroscopic results will essentially help to find the most reliable calibrations. In addition, apparent off-sets will be able to be identified.

Our last step will be to investigate the influence of the metallicity on open cluster parameter estimations that only take into account solar metallicity, and to provide correction values.

Acknowledgements. This work was supported by the financial contributions of the Austrian Agency for International Cooperation in Education and Research (WTZ CZ-11/2008, CZ-10/2010 and HR-14/2010), the City of Vienna (Hochschuljubiläumsstiftung project: H-1930/2008), the Forschungsstipendium der Universität Wien (F-416), a MOEL grant of the ÖFG (Project \#388), a travel grant from the Swedish Research Council and the Swedish National Space Board. This research has made use of the WEBDA database, operated at the Institute for Astronomy of the University of Vienna.

\section{References}

Ann, H. B., Lee, S. H., Sung, H., et al. 2002, AJ, 123, 905

Anthony-Twarog, B. J., \& Twarog, B. A. 2000, AJ, 119, 2282

Anthony-Twarog, B. J., \& Twarog, B. A. 2004, AJ, 127, 1000

Anthony-Twarog, B. J., \& Twarog, B. A. 2006, PASP, 118, 358 
Anthony-Twarog, B. J., Twarog, B. A., Laird, J. B., \& Payne, D. 1991, AJ, 101, 1902

Anthony-Twarog, B. J., Atwell, J., \& Twarog, B. A. 2005, AJ, 129, 872

Anthony-Twarog, B. J., Tanner, D., Cracraft, M., \& Twarog, B. A. 2006, AJ, 131, 461

Bessell, M. S. 1995, PASP, 107, 672

Bonatto, C., \& Bica, E. 2009, MNRAS, 394, 2127

Bruntt, H., Frandsen, S., Kjeldsen, H., \& Andersen, M. I. 1999, A\&AS, 140, 135

Cameron, L. M. 1985a, A\&A, 146, 59

Cameron, L. M. 1985b, A\&A, 147, 39

Canterna, R. 1976, AJ, 81, 228

Carney, B. W. 1979, ApJ, 233, 211

Cescutti, G., Matteucci, F., François, P., \& Chiappini, C. 2007, A\&A, 462, 943

Chen, L., Hou, J. L., \& Wang, J. J. 2003, AJ, 125, 1397

Chiappini, C., Matteucci, F., \& Romano, D. 2001, ApJ, 554, 1044

Clariá, J. J. 1982, A\&AS, 47, 323

Clariá, J. J. 1985, A\&AS, 59, 195

Clariá, J. J. 1986, A\&AS, 59, 195

Clariá, J. J., \& Lapasset, E. 1983, J. Astrophys. Astron., 4, 117

Clariá, J. J., \& Lapasset, E. 1985, MNRAS, 214, 229

Clariá, J. J., \& Lapasset, E. 1986a, ApJ, 302, 656

Clariá, J. J., \& Lapasset, E. 1986b, AJ, 91, 326

Clariá, J. J., \& Lapasset, E. 1989, MNRAS, 241, 301

Clariá, J. J., \& Mermilliod, J.-C. 1992, A\&AS, 95, 429

Clariá, J. J., \& Minniti, D. 1988, The Observatory, 108, 218

Clariá, J. J., Lapasset, E., \& Minniti, D. 1989, A\&AS, 78, 363

Clariá, J. J., Lapasset, E., \& Bosio, M. A. 1991, MNRAS, 249, 193

Clariá, J. J., Mermilliod, J.-C., Piatti, A. E., \& Minniti, D. 1994, A\&AS, 107, 39

Clariá, J. J., Piatti, A. E., \& Osborn, W. 1996, PASP, 108, 672

Clariá, J. J., Mermilliod, J.-C., \& Piatti, A. E. 1999, A\&AS, 134, 301

Clariá, J. J., Piatti, A. E., Lapasset, E., \& Mermilliod, J.-C. 2003, A\&A, 399, 543

Clariá, J. J., Piatti, A. E., Lapasset, E., \& Parisi, M. C. 2005, Baltic Astron., 14, 301

Clariá, J. J., Piatti, A. E., Parisi, M. C., \& Ahumada A. V. 2007, MNRAS, 379, 159

Clariá, J. J., Piatti, A. E., Mermilliod, J.-C., \& Palma, T. 2008, AN, 329, 609

Dawson, D. W. 1981, AJ, 86, 237

Dias, W. S., Alessi, B. S., Moitinho, A., \& Lepine, J. R. D. 2002, A\&A, 389, 871

Eggen, O. J. 1983, AJ, 88, 197

Geisler, D. P., \& Smith, V. V. 1984, PASP, 96, 871

Geisler, D. P., Clariá, J. J., \& Minniti, D. 1991, AJ, 102, 1836

Geisler, D. P., Clariá, J. J., \& Minniti, D. 1992, AJ, 104 ,1892

Girardi, L., Bressan, L., Bertelli, G., \& Chiosi, C. 2000, A\&AS, 141, 371

Groenewegen, M. A. T., Udalski, A., \& Bono, G. 2008, A\&A, 481, 441

Janes, K. A. 1975, ApJS, 29, 161

Janes, K. A. 1984, PASP, 96, 977

Janes, K. A., \& Smith, G. H. 1984, AJ, 89, 487

Jappsen, A.-K., Glover, S. C. O., Klessen, R. S., \& Mac Low, M.-M. 2007, ApJ, 660, 1332

Kaluzny, J., \& Mazur, B. 1991a, Acta Astron., 41, 167

Kaluzny, J., \& Mazur, B. 1991b, Acta Astron., 41, 191

Kaluzny, J., \& Mazur, B. 1991c, Acta Astron., 41, 279

Kaluzny, J., \& Rucinski, S. M. 1995, A\&AS, 114,

Kharchenko, N. V., Piskunov, A. E., Röser, S., Schilbach, E., \& Scholz, R.-D. 2005, A\&A, 438, 1163

Kharchenko, N. V., Piskunov, A. E., Röser, S., et al. 2009, A\&A, 504, 681

Kyeong, J., Kim, S. C., Hiriart, D., \& Sung, E.-C. 2008, J. Korean Astron. Soc., 41,147
Lallement, R., Welsh, B. Y., Vergely, J. L., Crifo, F., \& Sfeir, D. 2003, A\&A, 411,447

Luck, R. E., Kovtyukh, V. V., \& Andrievsky, S. M. 2006, AJ, 132, 902

Lynga, G., \& Wramdemark, S. 1984, A\&A, 132, 58

Machida, M. N. 2008, ApJ, 628, L1

Magrini, L., Sestito, P., Randich, S., \& Galli, D. 2009, A\&A, 494, 95

Martell, S., \& Laughlin, G. 2002, ApJ, 577, L45

McClure, R. D., \& van den Bergh, S. 1968, AJ, 73, 313

McClure, R. D., Twarog, B. A., \& Forrester, W. T. 1981, ApJ, 243, 841

Mermilliod, J.-C., Clariá, J. J., Andersen, J., Piatti, A. E., \& Mayor, M. 2001, A\&A, 375, 30

Nissen, P. E. 1980, IAUS, 85, 51

Nissen, P. E. 1988, A\&A, 199, 146

Nissen, P. E., Twarog, B. A., \& Crawford, D. L. 1987, AJ, 93, 634

Noriega-Mendoza, H., \& Ruelas-Mayorgo, A. 1997, AJ, 113, 722

Palous, J., \& Hauck, B. 1986, A\&A, 162, 54

Parisi, M. C., Clariá, J. J., Piatti, A. E., \& Geisler, D. 2005, MNRAS, 363, 1247

Pastoriza, M. G., \& Ropke, U. O. 1983, AJ, 88, 1769

Paunzen, E., \& Netopil, M. 2006, MNRAS, 371, 1641

Paunzen, E., Maitzen, H. M., Rakos, K. D., \& Schombert, J. 2003, A\&A, 403, 937

Paunzen, E., Stütz, Ch., \& Maitzen, H. M. 2005, A\&A, 441, 631

Pedicelli, S., Bono, G., \& Lemasle, B. et al. 2009, A\&A, 504, 81

Philip, A. G. D. 1976, Dudley Obs. Rep., 12, 1

Piatti, A. E., Clariá, J. J., \& Minniti D. 1993, J. Astrophys. Astron., 14, 145

Piatti, A. E., Clariá, J. J., \& Abadi, M. G. 1995, AJ, 110, 2813

Piatti, A. E., Clariá, J. J., \& Ahumada A. V. 2003a, MNRAS, 340, 1249

Piatti, A. E., Clariá, J. J., \& Ahumada A. V. 2003b, MNRAS, 346, 390

Piatti, A. E., Clariá, J. J., \& Ahumada A. V. 2004, A\&A, 418, 979

Piatti, A. E., Clariá, J. J., \& Ahumada A. V. 2006, MNRAS, 367, 599

Platais, I., Kozhurina-Platais, V., Barnes, S., et al. 2001, AJ, 122, 1486

Richtler, T. 1985, A\&AS, 59, 491

Sandage, A. 1969, ApJ, 158, 1115

Sanner, J., Geffert, M., Brunzendorf, J., \& Schmoll, J. 1999, A\&A, 349, 448 Schaller, G., Schaerer, G., Meynet, G., \& Maeder, A. 1992, A\&AS, 96, 269 Smith, G. H. 1983, PASP, 95, 296

Smith, G. H., \& Hesser, J. E. 1983, PASP, 95, 277

Smriglio, F., Dasgupta, A. K., Boyle, R. P., \& Nandy, K. 1990, A\&A, 228, 399

Straižys, V., \& Bartkevičius, A. 1982, Vilnius Astronomijos Observatorijos Biuletenis, 61, 22

Straižys, V., Kazlauskas, A., Cerniauskas, A., et al. 2003, Baltic Astron., 12, 323 Strömgren, B. 1966, ARA\&A, 4, 433

Sung, H., Bessell, M. S., Lee, B.-W., \& Lee, S.-G. 2002, AJ, 123, 290

Tadross A. L. 2001, New Astron., 6, 293

Tadross A. L. 2003, New Astron., 8, 737

Twarog, B. A. 1983, ApJ, 267, 207

Twarog, B. A., Anthony-Twarog, B. J., \& McClure, R. D. 1993, PASP, 105, 78

Twarog, B. A., Anthony-Twarog, B. J., \& Hawarden, T. G. 1995, PASP, 107, 1215

Twarog, B. A., Ashman, K. M., \& Anthony-Twarog, B. J. 1997, AJ, 114, 2556

Twarog, B. A., Corder, S., \& Anthony-Twarog, B. J. 2006, AJ, 132, 299

Twarog, B. A., Vargas, L. S., \& Anthony-Twarog, B. J. 2007, AJ, 134, 1777

Vansevicius, V., Platais, I., Paupers, O., \& Abolins, E. 1997, MNRAS, 285, 871

Welsh, B. Y., \& Shelton, R. L. 2009, Ap\&SS, 323, 1

Yong, D., Karakas, A. I., Lambert, D. L., Chieffi, A., \& Limongi, M. 2008, ApJ, 689, 1031

Pages 7 to 11 are available in the electronic edition of the journal at http://www . aanda.org 
E. Paunzen et al.: On the metallicity of open clusters. I.

Table 1. The $[\mathrm{Fe} / \mathrm{H}]$ values from photometric calibrations for open clusters found in the literature.

\begin{tabular}{|c|c|c|c|c|c|c|c|c|c|c|c|}
\hline Cluster & $\begin{array}{l}{[\mathrm{Fe} / \mathrm{H}]} \\
\end{array}$ & 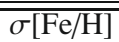 & Stars & System & Ref. & Cluster & {$[\mathrm{Fe} / \mathrm{H}]$} & 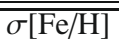 & Stars & System & Ref. \\
\hline Berkeley 1 & -0.14 & - & - & $\mathrm{J}$ & [57] & IC 4651 & +0.18 & 0.05 & 10 & $\mathrm{~S}$ & [41] \\
\hline Berkeley 2 & -0.59 & - & - & $\mathrm{J}$ & [57] & IC 4651 & -0.09 & 0.03 & - & $\mathrm{J}$ & [43] \\
\hline Berkeley 7 & -0.25 & - & - & $\mathrm{J}$ & [57] & IC 4651 & +0.08 & 0.09 & 8 & $\mathrm{D}$ & [49] \\
\hline Berkeley 14 & -1.20 & - & - & $\mathrm{J}$ & [57] & IC 4651 & -0.14 & - & - & $\mathrm{J}$ & [57] \\
\hline Berkeley 21 & -0.96 & - & - & $\mathrm{J}$ & [57] & IC 4651 & +0.095 & 0.053 & 11 & $\mathrm{D}$ & {$[61]$} \\
\hline Berkeley 22 & -0.42 & - & 7 & $\mathrm{~J}$ & [43] & IC 4665 & -0.11 & 0.07 & 5 & $\mathrm{~S}$ & [40] \\
\hline Berkeley 28 & -0.61 & - & - & $\mathrm{J}$ & [57] & IC 4725 & -0.092 & - & 63 & $\mathrm{~J}$ & [7] \\
\hline Berkeley 29 & -0.30 & - & 19 & $\mathrm{~J}$ & [43] & IC 4756 & +0.003 & - & 36 & $\mathrm{~J}$ & [7] \\
\hline Berkeley 30 & +0.10 & - & - & $\mathrm{J}$ & [57] & IC 4756 & +0.04 & 0.05 & 10 & $\mathrm{D}$ & [54] \\
\hline Berkeley 31 & -0.60 & - & - & $\mathrm{J}$ & [57] & IC 4756 & -0.012 & 0.072 & 6 & $\mathrm{D}$ & {$[61]$} \\
\hline Berkeley 32 & -0.37 & 0.05 & - & $\mathrm{J}, \mathrm{W}$ & [32] & IC 4996 & -0.47 & - & - & $\mathrm{J}$ & [57] \\
\hline Berkeley 32 & -0.37 & 0.04 & - & $\mathrm{J}$ & [43] & King 2 & -0.32 & - & - & $\mathrm{J}$ & [57] \\
\hline Berkeley 32 & -0.55 & - & - & $\mathrm{J}$ & [57] & King 7 & +0.03 & - & - & $\mathrm{J}$ & [57] \\
\hline Berkeley 39 & -0.42 & 0.11 & 8 & W & [29] & King 10 & +0.09 & - & - & $\mathrm{J}$ & [57] \\
\hline Berkeley 39 & -0.26 & - & 27 & $\mathrm{~J}$ & [43] & King 11 & -0.31 & - & 21 & $\mathrm{~J}$ & [43] \\
\hline Berkeley 39 & -0.33 & - & - & $\mathrm{J}$ & [57] & King 11 & -0.37 & - & - & $\mathrm{J}$ & [57] \\
\hline Berkeley 42 & +0.08 & - & - & $\mathrm{J}$ & [57] & Loden 807 & -0.19 & 0.06 & 2 & $\mathrm{D}$ & [25] \\
\hline Berkeley 58 & +0.09 & - & - & $\mathrm{J}$ & [57] & Loden 807 & -0.22 & - & 1 & D & [49] \\
\hline Berkeley 60 & -0.77 & - & - & $\mathrm{J}$ & [57] & Loden 807 & -0.130 & 0.090 & 1 & $\mathrm{D}$ & [61] \\
\hline Berkeley 62 & -0.85 & - & - & $\mathrm{J}$ & [57] & Melotte 20 & +0.07 & - & 119 & $\mathrm{~J}$ & [7] \\
\hline Berkeley 79 & -0.32 & - & - & $\mathrm{J}$ & [57] & Melotte 20 & +0.04 & 0.02 & 19 & $\mathrm{~S}$ & [40] \\
\hline Berkeley 86 & -0.08 & - & - & $\mathrm{J}$ & [57] & Melotte 20 & +0.01 & 0.09 & 19 & $\mathrm{~S}$ & [41] \\
\hline Collinder 74 & +0.05 & - & - & $\mathrm{J}$ & [57] & Melotte 22 & +0.08 & - & 31 & $\mathrm{~J}$ & [7] \\
\hline Collinder 140 & -0.092 & - & 21 & $\mathrm{~J}$ & [7] & Melotte 22 & +0.05 & 0.02 & 30 & $\mathrm{~S}$ & [40] \\
\hline Collinder 140 & +0.00 & 0.20 & - & $\mathrm{S}$ & [37] & Melotte 22 & +0.06 & 0.12 & 23 & $\mathrm{~S}$ & [41] \\
\hline Collinder 173 & -0.06 & 0.20 & - & $\mathrm{S}$ & [37] & Melotte 25 & +0.08 & - & 48 & $\mathrm{~J}$ & [7] \\
\hline Collinder 258 & +0.07 & 0.21 & 2 & $\mathrm{D}$ & [17] & Melotte 25 & +0.13 & 0.01 & 49 & $\mathrm{~S}$ & [40] \\
\hline Collinder 258 & -0.24 & - & 1 & $\mathrm{D}$ & [49] & Melotte 25 & +0.12 & 0.09 & 42 & $\mathrm{~S}$ & [41] \\
\hline Collinder 258 & -0.090 & 0.090 & 1 & D & [61] & Melotte 25 & +0.19 & 0.03 & 4 & $\mathrm{D}$ & [49] \\
\hline Collinder 272 & -0.40 & - & - & $\mathrm{J}$ & [57] & Melotte 25 & +0.175 & 0.034 & 4 & $\mathrm{D}$ & [61] \\
\hline Haffner 8 & -0.09 & 0.10 & 2 & $\mathrm{D}, \mathrm{W}$ & [17] & Melotte 66 & -0.35 & 0.20 & 4 & W & [28] \\
\hline Haffner 8 & +0.06 & 0.06 & 2 & D & [49] & Melotte 66 & -0.52 & 0.05 & - & $\mathrm{J}$ & [43] \\
\hline Haffner 8 & +0.060 & 0.040 & 2 & D & [61] & Melotte 66 & -0.44 & 0.09 & 6 & $\mathrm{D}$ & [49] \\
\hline IC 348 & +0.03 & - & - & $\mathrm{J}$ & [57] & Melotte 66 & -0.53 & 0.08 & - & $\mathrm{S}$ & {$[60]$} \\
\hline IC 1311 & -0.23 & - & - & $\mathrm{J}$ & [57] & Melotte 66 & -0.349 & 0.145 & 7 & D & [61] \\
\hline IC 1590 & -0.73 & - & - & $\mathrm{J}$ & [57] & Melotte 71 & -0.57 & 0.18 & 14 & W & [29] \\
\hline IC 1805 & +0.08 & - & - & $\mathrm{J}$ & [57] & Melotte 71 & -0.17 & 0.02 & - & C, $S$ & {$[62]$} \\
\hline IC 2391 & -0.15 & - & - & $\mathrm{S}$ & [27] & Melotte 105 & +0.00 & - & - & $\mathrm{J}$ & [57] \\
\hline IC 2391 & -0.09 & 0.20 & - & S & [37] & Melotte 111 & -0.063 & - & 30 & $\mathrm{~J}$ & [7] \\
\hline IC 2488 & +0.095 & 0.08 & 3 & $\mathrm{D}, \mathrm{W}$ & [22] & Melotte 111 & -0.03 & 0.02 & 18 & $\mathrm{~S}$ & [40] \\
\hline IC 2602 & -0.232 & - & - & $\mathrm{J}$ & [7] & Melotte 111 & -0.01 & 0.07 & 17 & $\mathrm{~S}$ & [41] \\
\hline IC 2714 & -0.12 & 0.09 & 19 & $\mathrm{D}, \mathrm{W}$ & [19] & NGC 103 & -0.85 & - & - & $\mathrm{J}$ & [57] \\
\hline IC 2714 & +0.01 & 0.10 & 10 & D & [49] & NGC 129 & -0.46 & - & - & $\mathrm{J}$ & [57] \\
\hline IC 2714 & -0.011 & 0.116 & 5 & D & [61] & NGC 188 & +0.055 & - & 34 & $\mathrm{~J}$ & [7] \\
\hline IC 4651 & +0.077 & 0.012 & 9 & S & [1] & NGC 188 & -0.16 & 0.11 & 7 & D & [49] \\
\hline IC 4651 & -0.194 & - & 96 & $\mathrm{~J}$ & [7] & NGC 188 & -0.046 & 0.098 & 8 & $\mathrm{D}$ & [61] \\
\hline IC 4651 & +0.21 & 0.03 & 31 & $\mathrm{D}$ & [10] & NGC 366 & -0.55 & - & - & $\mathrm{J}$ & [57] \\
\hline IC 4651 & +0.15 & 0.15 & - & W & [10] & NGC 381 & -0.04 & - & - & $\mathrm{J}$ & [57] \\
\hline IC 4651 & +0.11 & 0.05 & - & $\mathrm{D}, \mathrm{W}$ & [17] & NGC 433 & -0.68 & - & - & $\mathrm{J}$ & [57] \\
\hline NGC 436 & -0.77 & - & - & $\mathrm{J}$ & [57] & NGC 2301 & +0.060 & 0.060 & 2 & D & [61] \\
\hline NGC 457 & -0.46 & - & - & $\mathrm{J}$ & [57] & NGC 2324 & -0.163 & - & 64 & $\mathrm{~J}$ & [7] \\
\hline NGC 581 & -0.85 & - & - & $\mathrm{J}$ & [57] & NGC 2324 & -1.01 & 0.27 & 9 & W & [29] \\
\hline NGC 654 & -0.68 & - & - & $\mathrm{J}$ & [57] & NGC 2324 & -0.31 & 0.04 & 5 & W & [52] \\
\hline NGC 663 & -0.70 & - & - & $\mathrm{J}$ & [57] & NGC 2335 & +0.22 & - & - & D & [9] \\
\hline NGC 752 & -0.06 & 0.03 & 10 & $\mathrm{C}$ & [3] & NGC 2335 & +0.02 & - & 1 & D & [49] \\
\hline NGC 752 & -0.311 & - & 14 & $\mathrm{~J}$ & [7] & NGC 2335 & -0.030 & 0.090 & 1 & D & [61] \\
\hline NGC 752 & -0.26 & 0.06 & 7 & D & [20] & NGC 2343 & -0.15 & - & - & $\mathrm{D}$ & [9] \\
\hline NGC 752 & -0.02 & 0.02 & 29 & S & [40] & NGC 2343 & -0.39 & - & 1 & D & [49] \\
\hline NGC 752 & -0.05 & 0.13 & 26 & $\mathrm{~S}$ & [41] & NGC 2354 & -0.30 & 0.02 & 12 & $\mathrm{D}, \mathrm{J}, \mathrm{W}$ & [21] \\
\hline NGC 752 & -0.22 & 0.05 & 7 & D & [49] & NGC 2355 & +0.13 & - & 50 & $\mathrm{~J}$ & [34] \\
\hline NGC 752 & -0.21 & 0.09 & 19 & $\mathrm{D}, \mathrm{J}, \mathrm{S}$ & [58] & NGC 2355 & -0.14 & - & - & $\mathrm{J}$ & [57] \\
\hline NGC 752 & -0.160 & 0.040 & 6 & D & [61] & NGC 2360 & -0.092 & - & 34 & $\mathrm{~J}$ & [7] \\
\hline NGC 1039 & -0.291 & - & 36 & $\mathrm{~J}$ & [7] & NGC 2360 & -0.12 & 0.03 & 13 & D & [25] \\
\hline NGC 1193 & -0.45 & 0.12 & 32 & $\mathrm{~J}$ & [36] & NGC 2360 & -0.29 & 0.04 & 6 & W & [29] \\
\hline NGC 1193 & -0.54 & - & 16 & $\mathrm{~J}$ & [43] & NGC 2360 & -0.14 & 0.07 & 10 & D & [49] \\
\hline NGC 1342 & -0.163 & - & 14 & $\mathrm{~J}$ & [7] & NGC 2360 & -0.154 & 0.113 & 11 & D & [61] \\
\hline
\end{tabular}


Table 1. continued.

\begin{tabular}{|c|c|c|c|c|c|c|c|c|c|c|c|}
\hline Cluster & {$[\mathrm{Fe} / \mathrm{H}]$} & $\sigma[\mathrm{Fe} / \mathrm{H}]$ & $\overline{\text { Stars }}$ & $\overline{\text { System }}$ & $\overline{\text { Ref. }}$ & $\overline{\text { Cluster }}$ & {$[\mathrm{Fe} / \mathrm{H}]$} & $\sigma[\mathrm{Fe} / \mathrm{H}]$ & Stars & System & Ref. \\
\hline NGC 1342 & -0.24 & 0.12 & 3 & $\bar{D}$ & [49] & NGC 2420 & -0.37 & 0.05 & 106 & $\mathrm{C}, \mathrm{S}$ & [5] \\
\hline NGC 1528 & -0.133 & - & 17 & $\mathrm{~J}$ & {$[7]$} & NGC 2420 & -0.43 & 0.07 & - & $\mathrm{J}$ & [43] \\
\hline NGC 1545 & -0.16 & - & 1 & D & [20] & NGC 2420 & -0.43 & 0.10 & 8 & D & [49] \\
\hline NGC 1545 & -0.18 & - & 1 & D & [49] & NGC 2420 & -0.281 & 0.073 & 10 & D & [61] \\
\hline NGC 1545 & -0.060 & 0.090 & 1 & D & [61] & NGC 2422 & +0.11 & 0.10 & 11 & S & [41] \\
\hline NGC 1662 & -0.232 & - & 20 & $\mathrm{~J}$ & [7] & NGC 2423 & +0.00 & 0.08 & 4 & $\mathrm{D}, \mathrm{W}$ & [17] \\
\hline NGC 1662 & -0.13 & 0.02 & 2 & D & [20] & NGC 2423 & +0.03 & 0.03 & 8 & D & [25] \\
\hline NGC 1662 & -0.03 & 0.01 & 2 & D & [49] & NGC 2423 & +0.11 & 0.04 & 7 & D & [49] \\
\hline NGC 1662 & -0.095 & 0.015 & 2 & D & [61] & NGC 2423 & +0.143 & 0.092 & 3 & D & [61] \\
\hline NGC 1750 & -0.69 & - & - & $\mathrm{J}$ & [57] & NGC 2437 & -0.16 & 0.11 & 2 & D & [25] \\
\hline NGC 1798 & -0.46 & - & - & $\mathrm{J}$ & [57] & NGC 2437 & +0.07 & - & 1 & D & [49] \\
\hline NGC 1817 & -0.291 & - & 17 & $\mathrm{~J}$ & [7] & NGC 2447 & -0.10 & 0.08 & 9 & $\mathrm{D}, \mathrm{J}, \mathrm{W}$ & [23] \\
\hline NGC 1817 & -0.33 & 0.08 & 15 & W & [45] & NGC 2451 & -0.50 & 0.02 & - & $\mathrm{S}$ & [37] \\
\hline NGC 1931 & -0.06 & - & - & $\mathrm{J}$ & [57] & NGC 2451 & -0.106 & 0.122 & - & D & [46] \\
\hline NGC 2099 & +0.18 & 0.07 & 3 & D & [49] & NGC 2477 & -0.008 & - & 29 & $\mathrm{~J}$ & [7] \\
\hline NGC 2099 & +0.089 & 0.146 & 5 & D & [61] & NGC 2477 & -0.13 & 0.18 & 16 & W & [29] \\
\hline NGC 2112 & -1.30 & 0.22 & 4 & $\mathrm{~S}$ & [53] & NGC 2477 & +0.04 & 0.01 & - & D & [55] \\
\hline NGC 2158 & -0.36 & 0.18 & 3 & D & [49] & NGC 2477 & -0.05 & - & - & $\mathrm{J}$ & [57] \\
\hline NGC 2158 & -0.238 & 0.064 & 5 & D & [61] & NGC 2482 & +0.20 & 0.01 & - & D & [10] \\
\hline NGC 2168 & -0.28 & - & 1 & D & [49] & NGC 2482 & +0.10 & 0.10 & - & W & [10] \\
\hline NGC 2168 & -0.39 & - & - & $\mathrm{J}$ & [57] & NGC 2482 & +0.07 & 0.07 & 3 & $\mathrm{D}, \mathrm{W}$ & [17] \\
\hline NGC 2168 & -0.160 & 0.090 & 1 & D & [61] & NGC 2482 & +0.14 & 0.04 & 3 & D & [25] \\
\hline NGC 2192 & -0.22 & - & - & $\mathrm{J}$ & [57] & NGC 2482 & +0.13 & 0.04 & 3 & D & [49] \\
\hline NGC 2194 & -0.27 & 0.06 & 9 & W & [50] & NGC 2482 & +0.120 & 0.026 & 3 & D & [61] \\
\hline NGC 2204 & -0.618 & - & 9 & $\mathrm{~J}$ & {$[7]$} & NGC 2489 & +0.00 & - & 1 & D & [20] \\
\hline NGC 2204 & -0.41 & 0.19 & 6 & D & [26] & NGC 2489 & +0.10 & - & 1 & D & [49] \\
\hline NGC 2204 & -0.39 & 0.22 & 5 & D & [49] & NGC 2489 & +0.01 & - & - & $\mathrm{J}$ & [57] \\
\hline NGC 2204 & -0.338 & 0.250 & 5 & D & [61] & NGC 2489 & +0.080 & 0.010 & 2 & D & [61] \\
\hline NGC 2232 & -0.205 & 0.213 & - & D & [46] & NGC 2506 & -0.58 & 0.14 & 23 & W & [29] \\
\hline NGC 2236 & -0.30 & 0.20 & 13 & W & [24] & NGC 2506 & -0.57 & - & 16 & D & [38] \\
\hline NGC 2243 & -0.57 & 0.03 & 100 & $\mathrm{C}, \mathrm{S}$ & {$[4]$} & NGC 2506 & -0.48 & 0.08 & 4 & D & [49] \\
\hline NGC 2243 & -0.65 & 0.12 & 4 & $\mathrm{D}$ & [49] & NGC 2506 & -0.58 & - & - & $\mathrm{J}$ & [57] \\
\hline NGC 2243 & -0.480 & 0.160 & 5 & D & [61] & NGC 2506 & -0.368 & 0.108 & 5 & D & [61] \\
\hline NGC 2244 & -0.46 & - & - & $\mathrm{J}$ & [57] & NGC 2516 & -0.422 & - & 67 & $\mathrm{~J}$ & [7] \\
\hline NGC 2251 & -0.25 & 0.04 & 3 & W & [45] & NGC 2516 & +0.00 & 0.10 & 2 & D & [17] \\
\hline NGC 2251 & -0.17 & 0.06 & 3 & D & [49] & NGC 2516 & -0.28 & 0.20 & - & S & [37] \\
\hline NGC 2264 & +0.00 & - & - & D & [9] & NGC 2516 & +0.06 & 0.06 & 8 & $\mathrm{~S}$ & [41] \\
\hline NGC 2264 & -0.09 & 0.30 & - & $\mathrm{S}$ & [48] & NGC 2516 & +0.02 & - & 1 & D & [49] \\
\hline NGC 2264 & -0.16 & - & - & $\mathrm{J}$ & [57] & NGC 2516 & -0.10 & 0.04 & - & $\mathrm{J}$ & [56] \\
\hline NGC 2266 & -0.26 & 0.20 & 9 & W & [33] & NGC 2516 & +0.060 & 0.030 & 2 & D & [61] \\
\hline NGC 2281 & -0.074 & - & 38 & $\mathrm{~J}$ & [7] & NGC 2527 & -0.02 & - & - & D & [9] \\
\hline NGC 2287 & +0.065 & - & 14 & $\mathrm{~J}$ & [7] & NGC 2527 & -0.09 & - & 1 & D & [49] \\
\hline NGC 2287 & -0.10 & 0.11 & 10 & S & [41] & NGC 2527 & -0.080 & 0.090 & 1 & D & [61] \\
\hline NGC 2287 & -0.246 & 0.255 & - & D & [46] & NGC 2539 & +0.24 & 0.06 & - & D & [12] \\
\hline NGC 2287 & -0.13 & 0.06 & 3 & D & [49] & NGC 2539 & +0.00 & 0.20 & - & W & [12] \\
\hline NGC 2287 & +0.040 & 0.022 & 4 & D & [61] & NGC 2539 & +0.03 & 0.09 & 7 & $\mathrm{D}, \mathrm{W}$ & [17] \\
\hline NGC 2301 & +0.014 & - & 18 & $\mathrm{~J}$ & [7] & NGC 2539 & +0.17 & 0.08 & 3 & D & [49] \\
\hline NGC 2301 & +0.10 & 0.05 & 2 & D & [17] & NGC 2539 & +0.137 & 0.062 & 6 & D & [61] \\
\hline NGC 2301 & +0.04 & 0.12 & 13 & S & [41] & NGC 2546 & +0.26 & - & - & D & [9] \\
\hline NGC 2301 & +0.01 & - & 1 & D & [49] & NGC 2546 & +0.11 & +0.09 & 2 & D & [25] \\
\hline NGC 2546 & -0.14 & - & 1 & D & [49] & NGC 4755 & -0.21 & - & - & $\mathrm{J}$ & [57] \\
\hline NGC 2546 & +0.120 & 0.130 & 2 & D & [61] & NGC 4815 & -0.19 & - & - & $\mathrm{J}$ & [57] \\
\hline NGC 2547 & -0.121 & - & 43 & $\mathrm{~J}$ & [7] & NGC 5138 & +0.18 & - & 1 & $\mathrm{D}$ & [49] \\
\hline NGC 2547 & -0.13 & - & 1 & D & [8] & NGC 5138 & +0.120 & 0.050 & 2 & D & [61] \\
\hline NGC 2547 & -0.18 & 0.20 & - & $S$ & [37] & NGC 5168 & +0.05 & - & - & $\mathrm{J}$ & [57] \\
\hline NGC 2547 & -0.21 & - & 1 & D & [49] & NGC 5281 & -0.18 & - & 1 & $\mathrm{D}$ & [17] \\
\hline NGC 2547 & -0.160 & 0.090 & 1 & D & [61] & NGC 5316 & +0.19 & 0.11 & - & $\mathrm{D}, \mathrm{J}, \mathrm{W}$ & [14] \\
\hline NGC 2548 & +0.14 & - & - & D & [9] & NGC 5316 & -0.02 & 0.12 & 2 & $\mathrm{D}$ & [49] \\
\hline NGC 2548 & +0.01 & 0.02 & 2 & D & [49] & NGC 5316 & +0.128 & 0.127 & 4 & D & [61] \\
\hline NGC 2548 & +0.080 & 0.020 & 3 & D & [61] & NGC 5606 & +0.095 & - & - & $\mathrm{J}$ & [57] \\
\hline NGC 2567 & +0.00 & - & - & D & [13] & NGC 5617 & +0.31 & 0.10 & 2 & $\mathrm{D}, \mathrm{W}$ & [17] \\
\hline NGC 2567 & +0.00 & 0.10 & - & W & [13] & NGC 5617 & -0.32 & - & - & $\mathrm{J}$ & [57] \\
\hline NGC 2567 & -0.09 & 0.19 & 2 & $\mathrm{D}$ & [49] & NGC 5662 & -0.03 & 0.13 & 2 & $\mathrm{D}, \mathrm{W}$ & [18] \\
\hline NGC 2567 & +0.22 & - & - & $\mathrm{J}$ & [57] & NGC 5662 & +0.09 & - & - & $\mathrm{J}$ & [57] \\
\hline NGC 2567 & -0.030 & 0.120 & 2 & D & [61] & NGC 5822 & +0.01 & 0.04 & - & D & [11] \\
\hline NGC 2571 & +0.05 & - & 23 & $\mathrm{~J}$ & [7] & NGC 5822 & -0.25 & 0.15 & - & $\mathrm{W}$ & [11] \\
\hline
\end{tabular}


E. Paunzen et al.: On the metallicity of open clusters. I.

Table 1. continued.

\begin{tabular}{|c|c|c|c|c|c|c|c|c|c|c|c|}
\hline Cluster & {$[\mathrm{Fe} / \mathrm{H}]$} & $\sigma[\mathrm{Fe} / \mathrm{H}]$ & Stars & System & Ref. & Cluster & {$[\mathrm{Fe} / \mathrm{H}]$} & $\sigma[\mathrm{Fe} / \mathrm{H}]$ & Stars & System & $\overline{\text { Ref. }}$ \\
\hline NGC 2627 & -0.12 & 0.08 & 8 & W & {$[51]$} & NGC 5822 & -0.12 & 0.08 & 14 & $\mathrm{D}, \mathrm{W}$ & {$[17]$} \\
\hline NGC 2627 & -0.04 & - & - & $\mathrm{J}$ & [57] & NGC 5822 & +0.03 & 0.09 & 13 & D & [49] \\
\hline NGC 2632 & +0.04 & - & 76 & $J$ & [7] & NGC 5822 & -0.15 & 0.015 & - & $\mathrm{D}, \mathrm{J}$ & [59] \\
\hline NGC 2632 & +0.20 & 0.07 & 3 & D & [20] & NGC 5822 & -0.020 & 0.086 & 14 & D & [61] \\
\hline NGC 2632 & +0.09 & 0.02 & 47 & S & [40] & NGC 6025 & +0.195 & - & 48 & $\mathrm{~J}$ & [7] \\
\hline NGC 2632 & +0.10 & 0.15 & 42 & $\mathrm{~S}$ & [41] & NGC 6067 & +0.10 & 0.06 & 6 & $\mathrm{D}, \mathrm{W}$ & [17] \\
\hline NGC 2632 & +0.19 & 0.06 & 3 & D & [49] & NGC 6067 & -0.01 & 0.07 & 3 & D & [49] \\
\hline NGC 2632 & +0.142 & 0.069 & 4 & D & [61] & NGC 6067 & +0.138 & 0.064 & 5 & D & [61] \\
\hline NGC 2658 & -0.94 & - & - & $\mathrm{J}$ & [57] & NGC 6087 & -0.04 & - & - & $\mathrm{J}$ & [57] \\
\hline NGC 2660 & -1.05 & 0.16 & 7 & W & [29] & NGC 6134 & +0.28 & 0.02 & - & S & [6] \\
\hline NGC 2660 & -0.27 & 0.13 & 5 & D & [49] & NGC 6134 & -0.05 & 0.12 & 17 & $\mathrm{D}, \mathrm{J}, \mathrm{W}$ & [15] \\
\hline NGC 2660 & -0.05 & - & - & $\mathrm{J}$ & [57] & NGC 6134 & +0.20 & 0.10 & 7 & D & [49] \\
\hline NGC 2660 & -0.181 & 0.118 & 5 & D & [61] & NGC 6134 & +0.30 & - & - & $\mathrm{J}$ & [57] \\
\hline NGC 2669 & -0.20 & - & 1 & D & [17] & NGC 6134 & +0.182 & 0.087 & 10 & D & [61] \\
\hline NGC 2682 & -0.029 & - & 58 & $\mathrm{~J}$ & [7] & NGC 6192 & -0.10 & 0.09 & - & $\mathrm{S}$ & [47] \\
\hline NGC 2682 & -0.05 & 0.03 & 22 & D & [31] & NGC 6192 & -0.35 & - & - & $\mathrm{J}$ & [57] \\
\hline NGC 2682 & -0.06 & 0.07 & 36 & $\mathrm{~S}$ & [42] & NGC 6204 & -0.14 & - & - & $\mathrm{J}$ & [57] \\
\hline NGC 2682 & -0.05 & 0.04 & - & $\mathrm{J}$ & [43] & NGC 6208 & -0.03 & 0.06 & 3 & D & [49] \\
\hline NGC 2682 & -0.01 & 0.11 & 14 & D & [49] & NGC 6231 & +0.26 & - & - & $\mathrm{J}$ & [57] \\
\hline NGC 2682 & -0.11 & - & - & $\mathrm{J}$ & [57] & NGC 6259 & +0.06 & 0.08 & 8 & W & [39] \\
\hline NGC 2682 & +0.000 & 0.092 & 25 & D & [61] & NGC 6259 & +0.01 & 0.09 & 2 & $\mathrm{D}$ & [49] \\
\hline NGC 2818 & -0.31 & 0.13 & 11 & W & [29] & NGC 6281 & +0.07 & - & 37 & $\mathrm{~J}$ & [7] \\
\hline NGC 2818 & -0.29 & - & - & $\mathrm{J}$ & [57] & NGC 6281 & -0.08 & 0.08 & 2 & $\mathrm{D}, \mathrm{W}$ & [17] \\
\hline NGC 2910 & -0.04 & - & - & $\mathrm{J}$ & [57] & NGC 6281 & +0.00 & 0.12 & 2 & D & [49] \\
\hline NGC 2972 & -0.15 & 0.08 & 2 & D & [17] & NGC 6281 & +0.005 & 0.095 & 2 & D & [61] \\
\hline NGC 2972 & -0.09 & 0.01 & 2 & D & [49] & NGC 6405 & +0.07 & - & 20 & $\mathrm{~J}$ & [7] \\
\hline NGC 2972 & -0.073 & 0.021 & 3 & D & [61] & NGC 6425 & +0.25 & 0.04 & 2 & D & [25] \\
\hline NGC 3114 & -0.04 & 0.04 & 7 & $\mathrm{D}, \mathrm{W}$ & [17] & NGC 6425 & +0.09 & - & 1 & D & [49] \\
\hline NGC 3114 & -0.14 & 0.14 & 4 & D & [49] & NGC 6451 & -0.34 & 0.08 & - & S & [47] \\
\hline NGC 3114 & +0.022 & 0.070 & 5 & D & [61] & NGC 6451 & -0.20 & - & - & $\mathrm{J}$ & [57] \\
\hline NGC 3532 & +0.08 & 0.08 & 5 & W & [16] & NGC 6475 & +0.02 & 0.07 & 11 & $S$ & [41] \\
\hline NGC 3532 & -0.10 & 0.09 & 3 & D & [49] & NGC 6475 & +0.03 & - & 1 & D & [49] \\
\hline NGC 3532 & -0.022 & 0.088 & 5 & D & [61] & NGC 6475 & +0.070 & 0.090 & 1 & D & [61] \\
\hline NGC 3680 & -0.14 & 0.03 & 30 & $\mathrm{C}, \mathrm{S}$ & {$[2]$} & NGC 6494 & -0.14 & 0.15 & - & $\mathrm{D}, \mathrm{J}, \mathrm{W}$ & [14] \\
\hline NGC 3680 & +0.09 & 0.02 & - & S & [6] & NGC 6494 & +0.13 & 0.15 & 2 & $\mathrm{D}$ & [49] \\
\hline NGC 3680 & -0.145 & - & 27 & $\mathrm{~J}$ & [7] & NGC 6494 & +0.090 & 0.117 & 4 & D & [61] \\
\hline NGC 3680 & +0.00 & 0.04 & - & D & [10] & NGC 6520 & -0.25 & - & - & $\mathrm{J}$ & [57] \\
\hline NGC 3680 & +0.10 & 0.20 & - & W & [10] & NGC 6611 & -0.46 & - & - & $\mathrm{J}$ & [57] \\
\hline NGC 3680 & +0.02 & 0.03 & 8 & $\mathrm{D}, \mathrm{W}$ & [17] & NGC 6633 & -0.133 & - & 39 & $\mathrm{~J}$ & [7] \\
\hline NGC 3680 & +0.09 & 0.08 & 25 & $\mathrm{~S}$ & [41] & NGC 6633 & -0.01 & 0.03 & 5 & D & [25] \\
\hline NGC 3680 & -0.16 & 0.07 & 8 & D & [49] & NGC 6633 & -0.02 & 0.05 & 3 & $\mathrm{D}$ & [49] \\
\hline NGC 3680 & -0.121 & 0.051 & 8 & D & [61] & NGC 6649 & +0.05 & - & - & $\mathrm{J}$ & [57] \\
\hline NGC 3766 & -0.47 & - & - & $\mathrm{J}$ & [57] & NGC 6705 & +0.07 & - & 52 & $\mathrm{~J}$ & [7] \\
\hline NGC 3960 & -0.68 & 0.28 & 11 & W & [29] & NGC 6705 & -0.39 & - & - & $\mathrm{J}$ & [57] \\
\hline NGC 3960 & -0.06 & 0.05 & 3 & $\mathrm{D}$ & [49] & NGC 6716 & -0.311 & - & 24 & $\mathrm{~J}$ & [7] \\
\hline NGC 3960 & -0.170 & 0.131 & 6 & D & [61] & NGC 6716 & -0.26 & - & - & $\mathrm{J}$ & [57] \\
\hline NGC 4103 & -0.47 & - & - & $\mathrm{J}$ & [57] & NGC 6755 & -0.03 & - & - & $\mathrm{J}$ & [57] \\
\hline NGC 4349 & -0.23 & 0.08 & - & $\mathrm{D}, \mathrm{J}, \mathrm{W}$ & [14] & NGC 6791 & -0.08 & 0.07 & 7 & $\mathrm{D}$ & [30] \\
\hline NGC 4349 & -0.12 & 0.04 & 5 & D & [49] & NGC 6791 & +0.50 & - & - & $\mathrm{J}$ & [35] \\
\hline NGC 4349 & -0.060 & 0.123 & 6 & D & [61] & NGC 6791 & +0.05 & 0.05 & - & $\mathrm{J}$ & [43] \\
\hline NGC 4609 & +0.05 & 0.13 & 1 & $\mathrm{D}, \mathrm{W}$ & [17] & NGC 6791 & +0.00 & 0.14 & 6 & D & [49] \\
\hline NGC 6791 & +0.094 & 0.141 & 7 & D & [61] & Pismis 4 & -0.20 & 0.08 & 2 & D & [49] \\
\hline NGC 6791 & +0.45 & 0.04 & - & $\mathrm{C}, \mathrm{S}$ & [63] & Pismis 4 & -0.055 & 0.095 & 2 & D & [61] \\
\hline NGC 6823 & +0.08 & - & - & $\mathrm{J}$ & [57] & Pismis 20 & +0.03 & - & - & $\mathrm{J}$ & [57] \\
\hline NGC 6871 & -0.33 & - & - & $\mathrm{J}$ & [57] & Ruprecht 18 & +0.02 & - & - & $\mathrm{D}$ & [9] \\
\hline NGC 6913 & -0.55 & - & - & $\mathrm{J}$ & [57] & Ruprecht 18 & +0.08 & - & 1 & $\mathrm{D}$ & [49] \\
\hline NGC 6940 & +0.014 & - & 28 & $\mathrm{~J}$ & {$[7]$} & Ruprecht 18 & -0.010 & 0.090 & 1 & D & [61] \\
\hline NGC 7044 & +0.01 & - & - & $\mathrm{J}$ & [57] & Ruprecht 20 & -0.29 & 0.04 & 4 & $\mathrm{D}, \mathrm{W}$ & [17] \\
\hline NGC 7082 & +0.003 & - & 52 & $\mathrm{~J}$ & [7] & Ruprecht 32 & -0.38 & - & - & $\mathrm{J}$ & [57] \\
\hline NGC 7092 & +0.01 & 0.01 & 60 & S & [44] & Ruprecht 46 & -0.22 & - & - & D & [9] \\
\hline NGC 7209 & -0.01 & 0.11 & 2 & $\mathrm{D}$ & [20] & Ruprecht 46 & -0.12 & 0.09 & 2 & $\mathrm{D}$ & [17] \\
\hline NGC 7209 & -0.12 & - & 1 & D & [49] & Ruprecht 46 & -0.13 & 0.18 & 2 & $\mathrm{D}$ & [49] \\
\hline NGC 7209 & +0.070 & 0.050 & 2 & D & [61] & Ruprecht 79 & -0.14 & - & - & $\mathrm{J}$ & [57] \\
\hline NGC 7209 & -0.07 & 0.03 & 30 & V & [64] & Ruprecht 97 & -0.59 & - & 1 & D & [20] \\
\hline NGC 7235 & -0.62 & - & - & $\mathrm{J}$ & [57] & Ruprecht 97 & -0.03 & 0.03 & 2 & W & [25] \\
\hline NGC 7380 & -0.42 & - & - & $\mathrm{J}$ & [57] & Stock 2 & -0.14 & 0.20 & 2 & D & [20] \\
\hline
\end{tabular}


Table 1. continued.

\begin{tabular}{cccccc|cccccc}
\hline \hline Cluster & {$[\mathrm{Fe} / \mathrm{H}]$} & $\sigma[\mathrm{Fe} / \mathrm{H}]$ & Stars & System & Ref. & Cluster & {$[\mathrm{Fe} / \mathrm{H}]$} & $\sigma[\mathrm{Fe} / \mathrm{H}]$ & Stars & System & Ref. \\
\hline NGC 7419 & +0.05 & - & - & J & {$[57]$} & Tombaugh 2 & -0.60 & 0.18 & - & J & {$[43]$} \\
NGC 7789 & -0.24 & 0.13 & 17 & D & {$[49]$} & Trumpler 1 & -0.71 & - & - & J & {$[57]$} \\
NGC 7789 & -0.089 & 0.152 & 20 & D & {$[61]$} & Trumpler 10 & -0.13 & 0.20 & - & S & {$[37]$} \\
NGC 7790 & -0.22 & - & - & J & {$[57]$} & Trumpler 11 & -0.61 & - & - & J & {$[57]$} \\
Pismis 4 & -0.08 & 0.09 & 2 & D, W & {$[17]$} & Trumpler 14 & -0.03 & - & - & J & {$[57]$} \\
\hline
\end{tabular}

Notes. Photometric systems: Caby (C) DDO (D) Johnson (J) Strömgren (S) Vilnius (V) Washington (W).

Literature: [1] Anthony-Twarog \& Twarog (2000); [2] Anthony-Twarog \& Twarog (2004); [3] Anthony-Twarog \& Twarog (2006); [4] Anthony-Twarog et al. (2005); [5] Anthony-Twarog et al. (2006); [6] Bruntt et al. (1999); [7] Cameron (1985b); [8] Clariá (1982); [9] Clariá (1985); [10] Clariá \& Lapasset (1983); [11] Clariá \& Lapasset (1985); [12] Clariá \& Lapasset (1986); [13] Clariá \& Lapasset (1986); [14] Clariá \& Lapasset (1989); [15] Claria \& Mermilliod (1992); [16] Clariá \& Minniti (1988); [17] Clariá et al. (1989); [18] Clariá et al. (1991); [19] Clariá et al. (1994); [20] Clariá et al. (1996); [21] Clariá et al. (1999); [22] Clariá et al. (2003); [23] Clariá et al. (2005); [24] Clariá et al. (2007); [25] Clariá et al. (2008); [26] Dawson (1981); [27] Eggen (1983); [28] Geisler \& Smith (1984); [29] Geisler et al. (1992); [30] Janes (1984); [31] Janes \& Smith (1984); [32] Kaluzny \& Mazur (1991a); [33] Kaluzny \& Mazur (1991b); [34] Kaluzny \& Mazur (1991c); [35] Kaluzny \& Rucinski (1995); [36] Kyeong et al. (2008); [37] Lynga \& Wramdemark (1984); [38] McClure et al. (1981); [39] Mermilliod et al. (2001); [40] Nissen (1980); [41] Nissen (1988); [42] Nissen et al. (1987); [43] Noriega-Mendoza \& Ruelas-Mayorgo (1997); [44] Palous \& Hauck (1986); [45] Parisi et al. (2005); [46] Pastoriza \& Ropke (1983); [47] Paunzen et al. (2003); [48] Philip (1976); [49] Piatti et al. (1995); [50] Piatti et al. (2003a); [51] Piatti et al. (2003b); [52] Piatti et al. (2004); [53] Richtler (1985); [54] Smith (1983); [55] Smith \& Hesser (1983); [56] Sung et al. (2002); [57] Tadross (2003); [58] Twarog (1983); [59] Twarog et al. (1993); [60] Twarog et al. (1995); [61] Twarog et al. (1997); [62] Twarog et al. (2006); [63] Twarog et al. (2007); [64] Vansevicius et al. (1997). 
E. Paunzen et al.: On the metallicity of open clusters. I.

Table 3. Unweighted averaged $[\mathrm{Fe} / \mathrm{H}]$ values for 188 Galactic open clusters, 88 of them have more than one measurement.

\begin{tabular}{|c|c|c|c|c|c|c|c|c|c|c|c|}
\hline Cluster & $\overline{\overline{[\mathrm{Fe}} / \mathrm{H}]}$ & 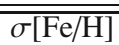 & $\overline{N N}$ & Cluster & $\overline{[\mathrm{Fe} / \mathrm{H}]}$ & 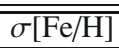 & $\overline{N N}$ & Cluster & {$[\mathrm{Fe} / \mathrm{H}]$} & $\sigma[\mathrm{Fe} / \mathrm{H}]$ & $N$ \\
\hline Berkeley 1 & -0.14 & 0.10 & 1 & NGC 1342 & -0.20 & 0.06 & 2 & NGC 4349 & -0.13 & 0.08 & 3 \\
\hline Berkeley 2 & -0.59 & 0.10 & 1 & NGC 1528 & -0.13 & 0.10 & 1 & NGC 4609 & +0.05 & 0.13 & 1 \\
\hline Berkeley 7 & -0.25 & 0.10 & 1 & NGC 1545 & -0.13 & 0.07 & 3 & NGC 4755 & -0.21 & 0.10 & 1 \\
\hline Berkeley 14 & -1.20 & 0.10 & 1 & NGC 1662 & -0.11 & 0.08 & 4 & NGC 4815 & -0.19 & 0.10 & 1 \\
\hline Berkeley 21 & -0.96 & 0.10 & 1 & NGC 1750 & -0.69 & 0.10 & 1 & NGC 5138 & +0.15 & 0.04 & 2 \\
\hline Berkeley 22 & -0.42 & 0.10 & 1 & NGC 1798 & -0.46 & 0.10 & 1 & NGC 5168 & +0.05 & 0.10 & 1 \\
\hline Berkeley 28 & -0.61 & 0.10 & 1 & NGC 1817 & -0.31 & 0.03 & 2 & NGC 5281 & -0.18 & 0.10 & 1 \\
\hline Berkeley 29 & -0.30 & 0.10 & 1 & NGC 1931 & -0.06 & 0.10 & 1 & NGC 5316 & +0.11 & 0.10 & 3 \\
\hline Berkeley 30 & +0.10 & 0.10 & 1 & NGC 2099 & +0.14 & 0.06 & 2 & NGC 5606 & +0.09 & 0.10 & 1 \\
\hline Berkeley 31 & -0.60 & 0.10 & 1 & NGC 2112 & -1.30 & 0.22 & 1 & NGC 5617 & +0.10 & 0.60 & 2 \\
\hline Berkeley 32 & -0.42 & 0.09 & 3 & NGC 2158 & -0.29 & 0.09 & 2 & NGC 5662 & +0.03 & 0.09 & 2 \\
\hline Berkeley 39 & -0.33 & 0.07 & 3 & NGC 2168 & -0.27 & 0.12 & 3 & NGC 5822 & -0.07 & 0.10 & 6 \\
\hline Berkeley 42 & +0.08 & 0.10 & 1 & NGC 2192 & -0.22 & 0.10 & 1 & NGC 6025 & +0.19 & 0.10 & 1 \\
\hline Berkeley 58 & +0.09 & 0.10 & 1 & NGC 2194 & -0.27 & 0.06 & 1 & NGC 6067 & +0.08 & 0.07 & 3 \\
\hline Berkeley 60 & -0.77 & 0.10 & 1 & NGC 2204 & -0.43 & 0.11 & 4 & NGC 6087 & -0.04 & 0.10 & 1 \\
\hline Berkeley 62 & -0.85 & 0.10 & 1 & NGC 2232 & -0.20 & 0.21 & 1 & NGC 6134 & +0.20 & 0.12 & 5 \\
\hline Berkeley 79 & -0.32 & 0.10 & 1 & NGC 2236 & -0.30 & 0.20 & 1 & NGC 6192 & -0.21 & 0.19 & 2 \\
\hline Berkeley 86 & -0.08 & 0.10 & 1 & NGC 2243 & -0.57 & 0.08 & 3 & NGC 6204 & -0.14 & 0.10 & 1 \\
\hline Collinder 74 & +0.05 & 0.10 & 1 & NGC 2244 & -0.46 & 0.10 & 1 & NGC 6208 & -0.03 & 0.06 & 1 \\
\hline Collinder 140 & -0.05 & 0.06 & 2 & NGC 2251 & -0.21 & 0.06 & 2 & NGC 6231 & +0.26 & 0.10 & 1 \\
\hline Collinder 173 & -0.06 & 0.20 & 1 & NGC 2264 & -0.08 & 0.08 & 3 & NGC 6259 & +0.04 & 0.04 & 2 \\
\hline Collinder 258 & -0.07 & 0.16 & 3 & NGC 2266 & -0.26 & 0.20 & 1 & NGC 6281 & +0.00 & 0.06 & 4 \\
\hline Collinder 272 & -0.40 & 0.10 & 1 & NGC 2281 & -0.07 & 0.10 & 1 & NGC 6405 & +0.07 & 0.10 & 1 \\
\hline Haffner 8 & +0.02 & 0.08 & 3 & NGC 2287 & -0.06 & 0.13 & 5 & NGC 6425 & +0.18 & 0.12 & 2 \\
\hline IC 348 & +0.03 & 0.10 & 1 & NGC 2301 & +0.05 & 0.04 & 5 & NGC 6451 & -0.27 & 0.10 & 2 \\
\hline IC 1311 & -0.23 & 0.10 & 1 & NGC 2324 & -0.38 & 0.39 & 3 & NGC 6475 & +0.04 & 0.03 & 3 \\
\hline IC 1590 & -0.73 & 0.10 & 1 & NGC 2335 & +0.08 & 0.15 & 3 & NGC 6494 & +0.04 & 0.13 & 3 \\
\hline IC 1805 & +0.08 & 0.10 & 1 & NGC 2343 & -0.25 & 0.17 & 2 & NGC 6520 & -0.25 & 0.10 & 1 \\
\hline IC 2391 & -0.12 & 0.04 & 2 & NGC 2354 & -0.30 & 0.02 & 1 & NGC 6611 & -0.46 & 0.10 & 1 \\
\hline IC 2488 & +0.09 & 0.08 & 1 & NGC 2355 & +0.02 & 0.20 & 2 & NGC 6633 & -0.05 & 0.06 & 3 \\
\hline IC 2602 & -0.23 & 0.10 & 1 & NGC 2360 & -0.15 & 0.07 & 5 & NGC 6649 & +0.05 & 0.10 & 1 \\
\hline IC 2714 & -0.04 & 0.07 & 3 & NGC 2420 & -0.38 & 0.07 & 4 & NGC 6705 & -0.10 & 0.36 & 2 \\
\hline IC 4651 & +0.07 & 0.13 & 10 & NGC 2422 & +0.11 & 0.10 & 1 & NGC 6716 & -0.28 & 0.03 & 2 \\
\hline IC 4665 & -0.11 & 0.07 & 1 & NGC 2423 & +0.08 & 0.07 & 4 & NGC 6755 & -0.03 & 0.10 & 1 \\
\hline IC 4725 & -0.09 & 0.10 & 1 & NGC 2437 & -0.03 & 0.17 & 2 & NGC 6791 & +0.23 & 0.30 & 6 \\
\hline IC 4756 & +0.01 & 0.03 & 3 & NGC 2447 & -0.10 & 0.08 & 1 & NGC 6823 & +0.08 & 0.10 & 1 \\
\hline IC 4996 & -0.47 & 0.10 & 1 & NGC 2477 & -0.03 & 0.07 & 4 & NGC 6871 & -0.33 & 0.10 & 1 \\
\hline King 2 & -0.32 & 0.10 & 1 & NGC 2482 & +0.13 & 0.05 & 6 & NGC 6913 & -0.55 & 0.10 & 1 \\
\hline King 7 & +0.03 & 0.10 & 1 & NGC 2489 & +0.05 & 0.05 & 4 & NGC 6940 & +0.01 & 0.10 & 1 \\
\hline King 10 & +0.09 & 0.10 & 1 & NGC 2506 & -0.51 & 0.10 & 5 & NGC 7044 & +0.01 & 0.10 & 1 \\
\hline King 11 & -0.34 & 0.04 & 2 & NGC 2516 & -0.07 & 0.16 & 7 & NGC 7082 & +0.00 & 0.10 & 1 \\
\hline Loden 807 & -0.18 & 0.05 & 3 & NGC 2527 & -0.06 & 0.04 & 3 & NGC 7092 & +0.01 & 0.01 & 1 \\
\hline Melotte 20 & +0.04 & 0.03 & 3 & NGC 2539 & +0.12 & 0.10 & 5 & NGC 7209 & -0.03 & 0.08 & 4 \\
\hline Melotte 22 & +0.06 & 0.01 & 3 & NGC 2546 & +0.11 & 0.16 & 4 & NGC 7235 & -0.62 & 0.10 & 1 \\
\hline Melotte 25 & +0.14 & 0.04 & 5 & NGC 2547 & -0.16 & 0.04 & 5 & NGC 7380 & -0.42 & 0.10 & 1 \\
\hline Melotte 66 & -0.43 & 0.08 & 5 & NGC 2548 & +0.08 & 0.07 & 3 & NGC 7419 & +0.05 & 0.10 & 1 \\
\hline Melotte 71 & -0.33 & 0.31 & 2 & NGC 2567 & +0.03 & 0.14 & 5 & NGC 7789 & -0.15 & 0.11 & 2 \\
\hline Melotte 105 & +0.00 & 0.10 & 1 & NGC 2571 & +0.05 & 0.10 & 1 & NGC 7790 & -0.22 & 0.10 & 1 \\
\hline Melotte 111 & -0.03 & 0.03 & 3 & NGC 2627 & -0.08 & 0.06 & 2 & Pismis 4 & -0.11 & 0.07 & 3 \\
\hline NGC 103 & -0.85 & 0.10 & 1 & NGC 2632 & +0.13 & 0.06 & 6 & Pismis 20 & +0.03 & 0.10 & 1 \\
\hline NGC 129 & -0.46 & 0.10 & 1 & NGC 2658 & -0.94 & 0.10 & 1 & Ruprecht 18 & +0.03 & 0.05 & 3 \\
\hline NGC 188 & -0.04 & 0.11 & 3 & NGC 2660 & -0.27 & 0.32 & 4 & Ruprecht 20 & -0.29 & 0.04 & 1 \\
\hline NGC 366 & -0.55 & 0.10 & 1 & NGC 2669 & -0.20 & 0.10 & 1 & Ruprecht 32 & -0.38 & 0.10 & 1 \\
\hline NGC 381 & -0.04 & 0.10 & 1 & NGC 2682 & -0.04 & 0.03 & 7 & Ruprecht 46 & -0.15 & 0.06 & 3 \\
\hline NGC 433 & -0.68 & 0.10 & 1 & NGC 2818 & -0.30 & 0.02 & 2 & Ruprecht 79 & -0.14 & 0.10 & 1 \\
\hline NGC 436 & -0.77 & 0.10 & 1 & NGC 2910 & -0.04 & 0.10 & 1 & Ruprecht 97 & -0.22 & 0.48 & 2 \\
\hline NGC 457 & -0.46 & 0.10 & 1 & NGC 2972 & -0.10 & 0.04 & 3 & Stock 2 & -0.14 & 0.20 & 1 \\
\hline NGC 581 & -0.85 & 0.10 & 1 & NGC 3114 & -0.05 & 0.08 & 3 & Tombaugh 2 & -0.60 & 0.18 & 1 \\
\hline NGC 654 & -0.68 & 0.10 & 1 & NGC 3532 & -0.01 & 0.09 & 3 & Trumpler 1 & -0.71 & 0.10 & 1 \\
\hline NGC 663 & -0.70 & 0.10 & 1 & NGC 3680 & -0.02 & 0.11 & 9 & Trumpler 10 & -0.13 & 0.20 & 1 \\
\hline NGC 752 & -0.15 & 0.11 & 8 & NGC 3766 & -0.47 & 0.10 & 1 & Trumpler 11 & -0.61 & 0.10 & 1 \\
\hline NGC 1039 & -0.29 & 0.10 & 1 & NGC 3960 & -0.23 & 0.29 & 3 & Trumpler 14 & -0.03 & 0.10 & 1 \\
\hline NGC 1193 & -0.49 & 0.07 & 2 & NGC 4103 & -0.47 & 0.10 & 1 & & & & \\
\hline
\end{tabular}

